\title{
UPDATES ON 4-HYDROXYDERRICIN AND XANTHOANGELOL OF ANGELICA PLANTS: EXTRACTION AND PHARMACOLOGICAL ACTIVITIES
}

\author{
D. L. Aulifa ${ }^{1,2^{*}}$, I. K. Adnyana ${ }^{3}$, Sukrasno ${ }^{1}$ and J. Levita ${ }^{4}$ \\ ${ }^{1}$ Pharmaceutical Biology Research Group, School of Pharmacy, Bandung Institute of \\ Technology, Jl. Ganesha 10, Bandung 40132, Indonesia \\ ${ }^{2}$ Department of Pharmaceutical Biology, Indonesian School of Pharmacy (Sekolah Tinggi \\ Farmasi Indonesia), Jl. Soekarno-Hatta No 354, Bandung 40226, Indonesia \\ ${ }^{3}$ Department of Pharmacology and Toxicology, School of Pharmacy, Bandung Institute of \\ Technology, Jl. Ganesha 10, Bandung 40132, Indonesia \\ ${ }^{4}$ Department of Pharmacology and Clinical Pharmacy Faculty of Pharmacy, \\ Universitas Padjadjaran, Jl. Raya Bandung-Sumedang km. 21 West Java 45363, Indonesia \\ *E-mail: diahliaaulifa@stfi.ac.id
}

\begin{abstract}
Two chalcones, e.g. 4-hydroxyderricin and xanthoangelol, have been proven in revealing many potential pharmacological activities, therefore their extraction method is critical because it influences the yield and, furthermore, its pharmacological activities. This review highlights the extraction method and various pharmacological activities of 4-hydroxyderricin and xanthoangelol from Angelica keiskei plants. The chalcone's diverse pharmacological activities are attributed to the flexible conformation of the opened-C ring and the moderate lipophilicity character on the A aromatic ring. The presence of a double bond in conjugation with carbonyl moiety is also important. However, further explorations need to be performed to obtain a remarkable yield of these particular prenylated-compounds and to understand their mechanisms of actions.
\end{abstract}

Keywords: Angelica keiskei, Chalcones, 4-Hydroxyderricin, Xanthoangelol

(C) RASĀYAN. All rights reserved

\section{INTRODUCTION}

Chalcones (Fig-1a), are 1,3-diphenyl-2-propen-1-one flavonoids that may exist in cis and trans isomeric forms. In higher plants, chalcones are synthesized from malonyl CoA and cinnamic acid by chalcone synthase. ${ }^{1}$ The molecular structure of these compounds consists of two aromatic rings which are linked by $\alpha, \beta$-unsaturated ketone groups $(-\mathrm{CO}-\mathrm{CH}=\mathrm{CH}-)$, where various substituents are attached and form an almost linear or planar structure..$^{2-3}$ Chalcones, which have been empirically used to treat many diseases, are contained in fruits, vegetables, and various plants. ${ }^{4}$ For example, the root and the stem of Angelica keiskei (ashitaba) contains yellow exudate, in which 2 major chalcones (4-hydroxyderricin and xanthoangelol) and flavonoid compound (cynaroside) are produced. ${ }^{5-6}$

The aim of this review article is to highlight the current updates in the extraction method and the pharmacological researches of 4-hydroxyderricin (4-HD) and xanthoangelol, natural chalcones of Angelica plants. The cited literature was selected in the publication period between January 2000 and March 2019.

\section{EXPERIMENTAL}

Briefly, a literature search in ScopeMed database using the keywords "4-hydroxyderricin" AND "xanthoangelol" AND "chalcones" AND "angelica" [in Title] resulted in 4 articles; in PubMed database using medical subject headings (MeSH) terms and the keywords "chalcones" [MeSH Terms] OR "chalcones" [All Fields] OR "chalcone" [MeSH Terms] OR "chalcone" [All Fields]) AND "4Rasayan J. Chem., 13(1), 11-17(2020) http://dx.doi.org/10.31788/RJC.2020.1315397

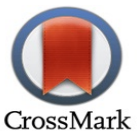


RASĀYAN J. Chem.

Vol. 13 | No. 1 |11 - 17| January - March | 2020

hydroxyderricin" [Supplementary Concept] OR "4-hydroxyderricin" [All Fields] OR "4 hydroxyderricin" [All Fields] AND "xanthoangelol" [Supplementary Concept] OR "xanthoangelol" [All Fields] AND "angelica" [MeSH Terms] OR "angelica" [All Fields] resulted in 28 articles; in Mendeley database using the keywords "4-hydroxyderricin" AND "xanthoangelol" AND "chalcones" AND "angelica" [in Papers] resulted in 63 articles; in GoogleScholar using the keywords "4-hydroxyderricin" AND "xanthoangelol" AND "chalcones" AND "angelica" [ranged between 2000-2019, include patents] resulted in 252 articles. Of those, articles excluded were: (1) published before year 2000; (2) Chalcones were not isolated from Angelica plants; (3) Review articles about chalcones; (4) Study for crops or others; (5) Duplicate articles found in different database; (6) Articles were not using English/Indonesian language; (7) Others.

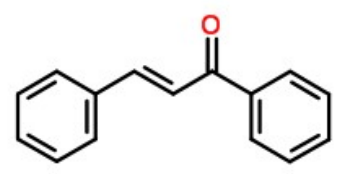

(a)

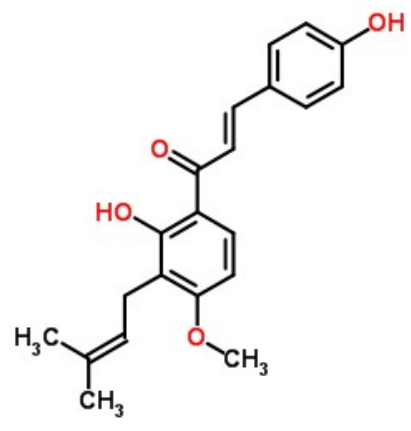

(b)

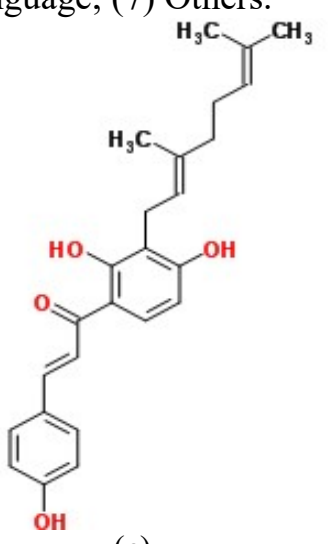

(c)

Fig.-1: (a) Chalcone $\mathrm{C}_{15} \mathrm{H}_{12} \mathrm{O}$ (ChemSpider ID 553346, http://www.chemspider.com/ChemicalStructure.553346.html accessed 01:47, Apr 10, 2019); (b) 4-hydroxyderricin $\mathrm{C}_{21} \mathrm{H}_{22} \mathrm{O}_{4}$ (ChemSpider ID 4942973, $\mathrm{http}: / /$ www.chemspider.com/Chemical-Structure.4942973.html, accessed 01:36, Apr 10, 2019); (c) xanthoangelol $\mathrm{C}_{25} \mathrm{H}_{28} \mathrm{O}_{4}$ (ChemSpider ID: 558181, http://www.chemspider.com/Chemical-Structure.558181.html accessed 01:50,

\section{Extraction Method} Apr 10, 2019)

Plants may contain secondary metabolites, which are distributed un-uniformly in the tissue, cellular and subcellular levels. ${ }^{7}$ Therefore, an important stage of analytical methods is the extraction step, as it greatly affects the type and quality of the analytical data. ${ }^{8}$ Medicinal plants study is usually initiated with the wet sortation of the specimen, the washing, the drying, and the grinding, prior to the extraction. The extraction itself is a separation method of active portions of the plant by using selective solvents through standard procedures. ${ }^{9}$ In this process, dissolves like is always employed in selecting the solvents. Polar solvents in aqueous mixtures containing methanol, ethanol, acetone, and ethyl acetate are often employed in extracting polyphenolic compounds from a plant matrix. ${ }^{10}$ All these stages are critical due to their effect on the outcomes (e.g. the yield and the content of phytoconstituents). Maceration, Reflux, and Soxhletextraction are commonly used in a laboratory-scale project. Recent extraction technique introduced microwave-assisted extraction (MAE), which is important for extracting valuable compounds from vegetal materials. ${ }^{11-13}$ MAE is quite adaptable on both laboratory and industry scale. ${ }^{14}$

An invention about the extraction process (drying, pulverizing, hot-dip extracting with ethanol) for Angelica chalcones in stems to obtain a liquid extract, had been registered. The ethanol extract was dissolved in water and extracted with ethyl acetate under reduced pressure. The crude chalcones were subjected into macro-porous adsorption column for separation and purification by column chromatography on silica gel to obtain chalcones (CN102020544B CN Grant, 2010). ${ }^{15}$

Different solvents employed to extract the chalcones from different parts of Angelica plants and their resulted-outcomes were provided in Table-1.

\section{Pharmacological Activities}

Many pharmacological activities are possessed by chalcones, e.g. (1) Suppresses the differentiation of pre-adipocytes to adipocytes via AMP-activated protein kinase and mitogen-activated protein kinases pathways; (2) Inhibits MAO; (3) Antioxidant and inhibits $\alpha$-glucosidase; (4) Stimulates GLUT4- 
RASĀYAN J. Chem.

Vol. 13 | No. 1 |11 - 17| January - March | 2020

dependent glucose; (5) Induces apoptosis in neuroblastoma and leukemia cells; (6) Antitumor-promotion; (6) PTP1B inhibitor; (7) Inhibits influenza virus neuraminidase; etc. A summary of Angelica chalcones' pharmacological activities is provided in Table-2. Each Angelica chalcone demonstrates multiple pharmacological activities.

Table-1: Extraction Method of Chalcones in Angelica Plants

\begin{tabular}{|c|c|c|c|c|}
\hline $\begin{array}{l}\text { Angelica } \\
\text { plant part }\end{array}$ & $\begin{array}{l}\text { Solvent used for } \\
\text { extraction }\end{array}$ & Chromatography system & Results & References \\
\hline $\begin{array}{l}\text { Dried stem } \\
\text { exudate }\end{array}$ & $\begin{array}{c}\text { A mixture of } n- \\
\text { hexane-MeOH- } \mathrm{H}_{2} \mathrm{O} \\
(95: 95: 10, \mathrm{v} / \mathrm{v} / \mathrm{v})\end{array}$ & $\begin{array}{l}\text { Chromatographed over } \\
\text { an octadecylsilane C18 } \\
\text { column }(2.5 \mathrm{~cm} \text { i.d. x } 50 \\
\text { cm; eluting solvent } \\
\text { MeOH; flow rate } 3.0 \\
\text { ml } / \mathrm{min})\end{array}$ & $\begin{array}{c}\text { Xanthoangelol } \\
(\text { yield }=2.4686 \%), \\
\text { xanthoangelol-F } \\
(\text { yield }=0.7099 \%), \\
\text { xanthoangelol-H (yield }=0.0284 \\
\%), \\
\text { isobavachalcone } \\
(\text { yield }=0.1744 \%), \\
\text { 4-hydroxyderricin }(\text { yield }= \\
1.4361 \%)\end{array}$ & 5 \\
\hline $\begin{array}{l}\text { Dried stem } \\
\text { exudate }\end{array}$ & $\begin{array}{c}\text { A mixture of } n- \\
\text { hexane-MeOH- } \mathrm{H}_{2} \mathrm{O} \\
(19: 19: 2, \mathrm{v} / \mathrm{v} / \mathrm{v})\end{array}$ & $\begin{array}{l}\text { Chromatographed over } \\
\text { an octadecylsilane C18 } \\
\text { column, continued with } \\
\text { preparative HPLC }\end{array}$ & $\begin{array}{c}\text { Xanthoangelol-I } \\
(\text { yield }=0.0135 \%), \\
\text { xanthoangelol-J } \\
(\text { yield }=0.0089 \%), \\
\text { deoxyxanthoangelol-H (yield = } \\
0.0762 \%) \\
\end{array}$ & 16 \\
\hline $\begin{array}{l}\text { Dried stem } \\
\text { exudate }\end{array}$ & Not described & Not described & $\begin{array}{c}\text { Xanthoangelol } \\
(\text { yield }=10.3 \%), \text { xanthoangelol-F } \\
(\text { yield }=2.44 \%), \\
\text { 4-hydroxyderricin }(\text { yield }= \\
9.39 \%), \\
\text { isobavachalcone } \\
\text { (yield }=0.134 \%), \text { xanthoangelol- } \\
\text { H (yield }=0.0049 \%), \\
\text { xanthoangelol-I }(\text { yield }= \\
0.0152 \%)\end{array}$ & 17 \\
\hline $\begin{array}{l}\text { Chopped } \\
\text { stem }\end{array}$ & $\begin{array}{l}\text { MeOH repeated } \\
\text { twice, followed by } \\
\text { fractionation using } \\
\text { of } n \text {-hexane and } \\
\text { EtOAc }\end{array}$ & $\begin{array}{l}\text { Chromatographed over a } \\
\text { silica gel column }(1.5 \mathrm{~cm} \\
\text { i.d. } \times 85 \mathrm{~cm}) \text { using } 20- \\
80 \% \text { EtOAc in hexane }\end{array}$ & $\begin{array}{c}\text { Xanthokeismin-A, } \\
\text { xanthokeismin-B, xanthokeismin- } \\
\text { C }\end{array}$ & 18 \\
\hline Seeds & $\begin{array}{l}\text { A mixture of of } n \text { - } \\
\text { hexane-MeOH- } \\
\mathrm{H}_{2} \mathrm{O} \text {. }\end{array}$ & $\begin{array}{l}\text { Chromatographed over } \\
\text { an octadecylsilane } \mathrm{C} 18 \\
\text { column using } \mathrm{MeOH} \text { in } \\
\mathrm{H}_{2} \mathrm{O} \text { gradient mixtures. }\end{array}$ & $\begin{array}{c}\text { Ashitabaol-A } \\
\text { (yield }=0.009 \%, \text { colorless } \\
\text { needles })\end{array}$ & 19 \\
\hline $\begin{array}{l}\text { Dried root } \\
\text { powder }\end{array}$ & $\begin{array}{l}\text { EtOH } 100 \% \text { for } 30 \\
\text { min under gentle } \\
\text { agitation; repeated } \\
\text { twice. }\end{array}$ & $\begin{array}{l}\text { Chromatographed over a } \\
\text { cosmosil140 C18-OPN. }\end{array}$ & $\begin{array}{l}\text { Xanthoangelol and } 4- \\
\text { hydroxyderricin both with purity } \\
>95 \%\end{array}$ & 20 \\
\hline $\begin{array}{l}\text { Dried root } \\
\text { powder }\end{array}$ & $\begin{array}{c}\text { A mixture of } n- \\
\text { hexane-MeOH- } \mathrm{H}_{2} \mathrm{O} \\
(19: 19: 2, \mathrm{v} / \mathrm{v} / \mathrm{v})\end{array}$ & $\begin{array}{l}\text { Chromatographed over a } \\
\text { silica gel column using } \\
\text { stepwise gradient elution } \\
\text { with } n \text {-hexane-EtOAc } \\
(9: 1 \rightarrow 0: 1 ; \text { EtOAc- } \\
\text { MeOH } 1: 0 \rightarrow 1: 4)\end{array}$ & $\begin{array}{c}\text { Xanthoangelol } \\
(\text { yield }=0.0411 \%), \\
\text { 4-hydroxyderricin }(\text { yield }= \\
0.0073 \%),\end{array}$ & 21 \\
\hline $\begin{array}{l}\text { Dried root } \\
\text { powder }\end{array}$ & $\begin{array}{c}\text { EtOH at room } \\
\text { temperature for } 30 \\
\text { min; repeated twice }\end{array}$ & $\begin{array}{l}\text { ODS chromatography } \\
\text { successively eluting with } \\
30,40,75,100 \% \text { EtOH. } \\
\text { The } 40 \% \text { EtOH elute }\end{array}$ & $\begin{array}{l}\text { Xanthoangelol } \\
\text { (yield }=0.72 \%)\end{array}$ & 22 \\
\hline
\end{tabular}


RASĀYAN J. Chem.

Vol. 13 | No. 1 |11 - 17| January - March | 2020

\begin{tabular}{|c|c|c|c|c|}
\hline & & $\begin{array}{l}\text { was subjected to silica } \\
\text { gel chromatography and } \\
\text { eluted with a gradient of } \\
\mathrm{CHCl}_{3}-\mathrm{MeOH} \\
(50: 1 \rightarrow 5: 1, \mathrm{v} / \mathrm{v})\end{array}$ & & \\
\hline $\begin{array}{c}\text { Dried aerial } \\
\text { parts }\end{array}$ & $\begin{array}{c}\text { A mixture of } \\
\mathrm{MeOH}-\mathrm{H}_{2} \mathrm{O}(7: 3, \\
\mathrm{v} / \mathrm{v}: 25 \mathrm{l}) \text { at } 80^{\circ} \mathrm{C} \\
\text { for } 4 \mathrm{~h} \text {; repeated } \\
\text { three times }\end{array}$ & $\begin{array}{l}\text { Chromatographed over a } \\
\text { silica gel column (Silica } \\
\text { gel } 60,70-230 \text { mesh) } \\
\text { using stepwise gradient } \\
\text { elution with hexane- } \\
\text { dichloromethane (1:3), } \\
\text { dichloromethane-MeOH } \\
\text { (30:1), dichloromethane- } \\
\text { MeOH (3:1) }\end{array}$ & $\begin{array}{c}\text { Xanthoangelol } \\
\text { (yield }=0.031 \% ; 99 \% \text { purity; } \\
\text { yellow needles), } \\
\text { 4-hydroxyderricin (yield = } \\
0.004 \% ; 98 \% \text { purity; yellow } \\
\text { powder) }\end{array}$ & 23 \\
\hline
\end{tabular}

Table-2: Pharmacological Activities of Angelica's Chalcones

\begin{tabular}{|c|c|c|c|}
\hline $\begin{array}{l}\text { Chalcone } \\
\text { Compound }\end{array}$ & $\begin{array}{l}\text { Angelica Plant } \\
\text { Part }\end{array}$ & Biological Activities & References \\
\hline \multirow[t]{14}{*}{ Xanthoangelol } & Herbs & $\begin{array}{l}\text { (1) Suppresses melanoma-genesis by targeting BRAF and } \\
\text { PI3K }\end{array}$ & 24 \\
\hline & & $\begin{array}{l}\text { (2) Suppresses the differentiation of pre-adipocytes to } \\
\text { adipocytes }\end{array}$ & 25 \\
\hline & & (3) Inhibits MAO & 23 \\
\hline & & (4) Antioxidant and inhibits $\alpha$-glucosidase & 26 \\
\hline & \multirow{2}{*}{ Stem exudate } & (1) Antiplatelet activity in vivo in mouse & 27 \\
\hline & & (2) Stimulates GLUT4-dependent glucose & 28 \\
\hline & & $\begin{array}{l}\text { (3) Attenuates protein and mRNA levels of inflammatory } \\
\text { enzymes (iNOS and COX-2) }\end{array}$ & 29 \\
\hline & & $\begin{array}{l}\text { (4) Reduces the serum LDL levels, total cholesterol and } \\
\text { triglyceride contents in the liver rats }\end{array}$ & 30 \\
\hline & & (5) Induces apoptosis in neuroblastoma and leukemia cells & 31 \\
\hline & Root & (1) Antitumor and antimetastatic activity & 32 \\
\hline & & $\begin{array}{l}\text { (2) Inhibits platelet aggregation on rabbits which induced } \\
\text { by collagen, platelet-activating factor, and phorbol 12- } \\
\text { myristate } 13 \text {-acetate }\end{array}$ & 33 \\
\hline & & (3) Induces adiponectin production in 3T3-L1 adipocytes & 22 \\
\hline & & (4) Antitumor and antimetastatic & 34 \\
\hline & Stem & PTP1B inhibitor & 35 \\
\hline Xanthoangelol-B & Root & Inhibits influenza virus neuraminidase & 36 \\
\hline Xanthoangelol-D & $\begin{array}{l}\text { Root } \\
\text { Stem }\end{array}$ & $\begin{array}{l}\text { Could suppressed Nuclear Factor- } \kappa \mathrm{B} \\
\text { PTP1B inhibitor }\end{array}$ & $\begin{array}{l}37 \\
35\end{array}$ \\
\hline $\begin{array}{l}\text { Xanthoangelol-E, } \\
\text { xanthoangelol-K, } \\
\text { xanthoangelol-M }\end{array}$ & Stem & PTP1B inhibitor & 35 \\
\hline \multirow{3}{*}{ Xanthoangelol-F } & Root & \multirow{3}{*}{$\begin{array}{c}\text { Induces apoptotic cell death in human leukemia cells } \\
\text { (HL60) } \\
\text { (1) Cancer chemopreventive effects } \\
\text { (2) PTP1B inhibitor }\end{array}$} & 21 \\
\hline & \multirow[t]{2}{*}{ Stem } & & 5 \\
\hline & & & 35 \\
\hline Xanthoangelol-G & Root & Inhibits influenza virus neuraminidase & 36 \\
\hline $\begin{array}{l}\text { Xanthoangelol-H/ } \\
\text { Jejuchalcone D }\end{array}$ & Root & $\begin{array}{l}\text { Induces apoptotic cell death in human leukemia cells } \\
\text { (HL60) }\end{array}$ & 21 \\
\hline Xanthoangelol-I & Root & Induces apoptotic cell death in human leukemia cells & 21 \\
\hline
\end{tabular}


RASĀYAN J. Chem.

Vol. 13 | No. 1 |11 - 17| January - March | 2020

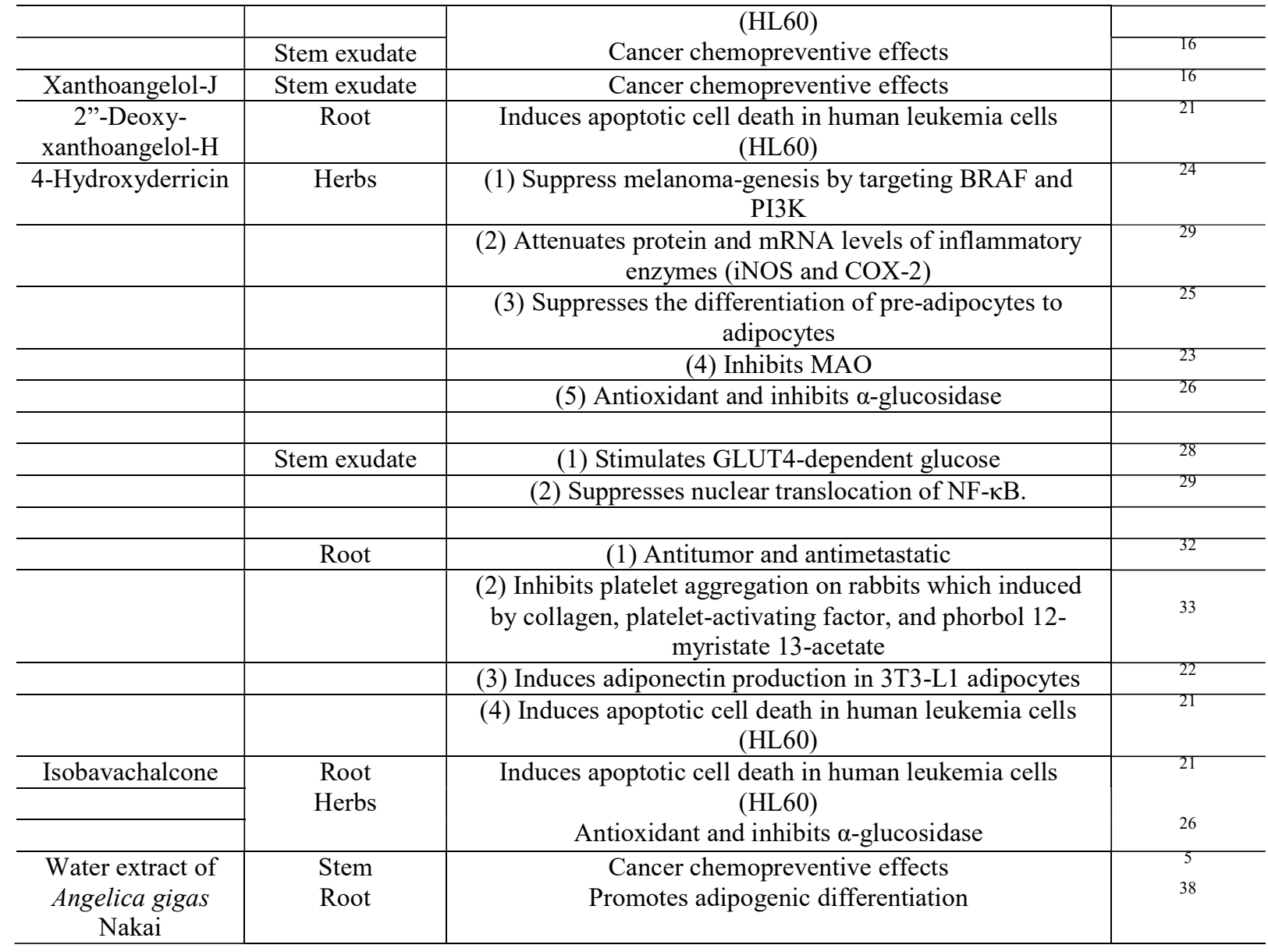

These diverse activities might be attributed to the flexible conformation of the backbone of the chalcone ${ }^{38-}$ 39; moreover, the long hydrocarbon bridge which attached to ring A of moderate hydrophobicity, contributes an important role for the pharmacological activity. ${ }^{42}$ The presence of a double bond in conjugation with carbonyl moiety of chalcones is also believed to be responsible for the pharmacological activities, as removal of this functionality makes them inactive. ${ }^{24}$

\section{CONCLUSION}

In this review, the extraction method of Angelica chalcones and their pharmacological activities have been summarized. Xanthoangelol and 4-HD, two major chalcones, are found abundantly in the stem exudate of Angelica plants. The varieties of solvents and chromatographic system for separating the chalcones from different parts of the plant at least answered the reason for their different yields. These chalcones have been proven exerting various pharmacological activities due to their specific conformation structures. However, these pharmacological activities might also be influenced by the selected extraction method. Further considerable works need to be performed to obtain a remarkable yield of the chalcones and to perceive the working mechanisms of chalcones-prenylated substances.

\section{REFERENCES}

1. N.J. Lawrence, R.P. Patterson, L.L. Ooi, D. Cook, and S. Ducki, Bioorganic and Medicinal Chemistry Letters, 16(22), 5844(2006), DOI: 10.1016/j.bmcl.2006.08.065

2. S.L. Soon, H.S. Kim, and D.U. Lee, Buletin-Korean Chemical Society, 28(12), 2495(2007), DOI: 10.5012/bkcs.2007.28.12.2495

3. S.K. Awasthi, N. Mishra, B. Kumar, M. Sharma, A. Bhattacharya, L.C. Mishra and V.K. Bashin, Medicinal Chemistry Research, 18(6), 407(2009), DOI: 10.1007/s00044-008-9137-9 
RASĀYAN J. Chem.

Vol. 13 | No. 1 |11 - 17| January - March | 2020

4. P. Singh, A. Anand, and V. Kumar, European Journal of Medicinal Chemistry, 85, 758 (2014), DOI: 10.1016/j.ejmech.2014.08.033

5. T. Akihisa, H. Tokuda, M. Ukiya, M. Iizuka, S. Schneider, K. Ogasawara, T. Mukainaka, K. Iwatsuki, T. Suzuki, and H. Nishino, Cancer Letters, 201(2), 133(2003), DOI: 0.1016/S03043835(03)00466-X

6. S. D. Sarker, and L. Nahar, Current Medicinal Chemistry, 11(11), 1479(2004), DOI: 10.2174/0929867043365189

7. P. Maisuthisakul, S. Pasuk, and P. Ritthiruangdej, Journal of Food Composition and Analysis, 21(3), 229(2008), DOI: 10.1016/j.jfca.2007.11.005

8. V. Camel, Encyclopedia of Analytical Chemistry: Application, Theory and Instrumentation, 1(2014), DOI: $10.1002 / 9780470027318 . a 9902$

9. G. Brusotti, I. Cesari, A. Dentamaro, G. Caccialanza, and G. Massolini, Journal of Pharmaceutical and Biomedical Analysis, 87, 218(2014), DOI: 10.1016/j.jpba.2013.03.007

10. B. Sultana, F. Anwar, and M. Ashraf, Molecules, 14(6), 2167(2009), DOI: 10.3390/molecules 14062167

11. J. G. Gujar, S. J. Wagh, and V. G. Gaikar, Separation and Purification Technology, 70(3), 257(2010), DOI: $10.1016 /$ j.seppur.2009.08.005

12. G. Spigno, and D. M. De Faveri, Journal of Food Engineering, 93(2), 210(2009), DOI: 10.1016/j.jfoodeng.2009.01.006

13. A. K. Babu, and K. Selvaraju, Rasayan Journal of Chemistry, 11(4), 1501(2018), DOI: 10.31788/RJC.2018.1144037

14. G. Cravotto, L. Boffa, S. Mantegna, P. Perego, M. Avogadro, and P. Cintas, Ultrasonics Sonochemistry, 15(5), 898(2008), DOI: 10.1016/j.ultsonch.2007.10.009

15. Z.C. Mĩn, Chinese Patent CN102020544B (2006).

16. T. Akihisa, H. Tokuda, D. Hasegawa, M. Ukiya, Y. Kimura, F. Enjo, T. Suzuki, H. Nishino, Journal of Natural Products, 69(1), 38(2006), DOI: 10.1021/np058080d

17. R. Nishimura, K. Tabata, M. Arakawa, Y. Ito, Y. Kimura, T. Akihisa, H. Nagai, A. Sakuma, H. Kohno, T. Suzuki, Biological and Pharmaceutical Bulletin, 30(10), 1878(2007), DOI: 10.1248/bpb.30.1878

18. N. Aoki, M. Muko, E. Ohta, and S. Ohta, Journal of Natural Products, 71(7), 1308(2008), DOI: $10.1021 / \mathrm{np} 800187 \mathrm{f}$

19. N. Aoki, and S. Ohta, Tetrahedron Letters, 51(26), 3449(2010), DOI: 10.1016/j.tetlet.2010.04.122

20. T. Enoki, H. Ohnogi, K. Nagamine, Y. Kudo, K. Sugiyama, M. Tanabe, E. Kobayashi, H. Sagawa, and I. Kato, Journal of Agricultural and Food Chemistry, 55(15), 6013(2007), DOI: $10.1021 / \mathrm{jf070720q}$

21. T. Akihisa, T. Kikuchi, H. Nagai, K. Ishii, K. Tabata and T. Suzuki, Journal of Oleo Science, 60(2), 71(2011), DOI: $10.5650 /$ jos.60.71

22. H. Ohnogi, Y. Kudo, K. Tahara, K. Sugiyama, T. Enoki, S. Hayami, H. Sagawa, Y. Tanimura, W. Aoi, Y. Naito, I. Kato, and T. Yoshikawa, Bioscience, Biotechnology and Biochemistry, 76(5), 961(2012), DOI: 10.1271/bbb.110976

23. J. H. Kim, Y. K. Son, G. H. Kim, and K. H. Hwang, Biomolecules and Therapeutics, 21(3), 234(2013), DOI: 10.4062/biomolther.2012.100

24. T. Zhang, Q. Wang, M. Fredimoses, G. Gao, K. Wang, H. Chen, T. Wang, N Oi, T. A. Zykova, K. Reddy, K. Yao, W. Ma, X. Chang, M.H. Lee, M.G. Rathore, A.M. Bode, H. Ashida, Z. Dong, Cancer Prevention Research, 11(10), 607(2018), DOI: 10.1158/1940-6207.CAPR-18-0092

25. T. Zhang, K. Sawada, N. Yamamoto, and H. Ashida, Molecular Nutrition and Food Research, 57(10), 1729(2013), DOI: 10.1002/mnfr.201300020

26. L. Luo, R. Wang, X. Wang, Z. Ma, and N. Li, Food Chemistry, 131(3), 992(2012), DOI: 10.1016/j.foodchem.2011.09.099

27. N. Ohkura, K. Ohnishi, M. Taniguchi, A. Nakayama, Y. Usuba, M. Fujita, A. Fujii, K. Ishibashi, K. Baba, G. Atsumi, Pharmazie, 71(11), 651(2016), DOI: 10.1691/ph.2016.6678

28. M. Ohta, A. Fujinami, N. Kobayashi, A. Amano, A. Ishigami, H. Tokuda, N. Suzuki, F. Ito, T. Mori, 
RASĀYAN J. Chem.

Vol. 13 | No. 1 |11 - 17| January - March | 2020

M. Sawada, K. Iwasa, J. Kitawaki, K. Ohnishi, M. Tsujikawa, H. Obayashi, Nutrition Research, 35(7), 618(2015), DOI: 10.1016/j.nutres.2015.05.010

29. H. R. Chang, H. J. Lee, and J.H. Ryu, Journal of Medicinal Food, 17(12), 1306(2014), DOI: 10.1089/jmf.2013.3037

30. H. Ogawa, Y. Okada, T. Kamisako, and K. Baba, Clinical and experimental pharmacology \& physiology, 34, 238(2007), DOI: 10.1111/j.1440-1681.2005.04147.x

31. K. Tabata, K. Motani, N. Takayanagi, R. Nishimura, S. Asami, Y. Kimura, M. Ukiya, D. Hasegawa, T. Akihisa, and T. Suzuki, Biological and Pharmaceutical Bulletin, 28(8), 1404(2005), DOI: $10.1248 / \mathrm{bpb} .28 .1404$

32. M. Sumiyoshi, M. Taniguchi, K. Baba, and Y. Kimura, Phytomedicine 22(7-8), 759(2015), DOI: 10.1016/j.phymed.2015.05.005

33. D.J. Son, Y.O. Park, C. Yu, S.E. Lee, and Y.H. Park, Natural Product Research, 28(24), 2312(2014), DOI: $10.1080 / 14786419.2014 .931389$

34. Y. Kimura, and K. Baba, International Journal of Cancer, 106(3), 429(2003), DOI: $10.1002 / \mathrm{ijc} .11256$

35. J.L. Li, L.X. Gao, F.W. Meng, C.L. Tang, R.J. Zhang, J.Y. Li, C. Luo, J. Li, W.M. Zhao, Bioorganic and Medicinal Chemistry Letters, 25(10), 2028(2015), DOI: 10.1016/j.bmcl.2015.04.003

36. J.Y. Park, H.J. Jeong, Y.M. Kim, S.J. Park, M.C. Rho, K.H. Park, Y.B. Ryu, W.S. Lee, Bioorganic and Medicinal Chemistry Letters, 21(18), 5602(2011), DOI: 10.1016/j.bmcl.2011.06.130

37. M. Sugii, M. Ohkita, M. Taniguchi, K. Baba, Y. Kawai, C. Tahara, M. Takaoka, and Y. Matsumura, Biological and Pharmaceutical Bulletin, 28(4), 607(2005), DOI: 10.1248/bpb.28.607

38. J.Y. Park, J. A Ko, D.W. Kim, Y.M. Kim, H.J. Kwon, H.J. Jeong, C.Y. Kim, K.H. Park, W.S. Lee, and Y.B. Ryu, Journal of Enzyme Inhibition and Medicinal Chemistry, 31(1), 23(2016), DOI: $10.3109 / 14756366.2014 .1003215$

39. B. Zhou, and C. Xing, Medicinal Chemistry, 5(8), 388(2015), DOI: 10.4172/21610444.1000291.Diverse

40. L.K. Caesar, and N.B. Cech, Planta Medica, 82(14), 1236(2016), DOI: 10.1055/s-0042-110496

41. A. Solankee, G. Patel and S. Solankee, Rasayan Journal of Chemistry, 1(3), 591(2008)

42. N. Ohkura, H. Oiwa, K. Ohnishi, M. Taniguchi, K. Baba, G.I. Atsumi, Journal of Intercultural Ethnopharmacology, 4(4), 355(2015), DOI: 10.5455/jice.20150910123122

[RJC-5397/2019] 